\title{
APPENDIX tored
}

\section{Ms. II G10792 TITLE: No evidence for Cretaceous-Tertiary boundary-age deep water deposits in the Caribbean and Gulf of Mexico}

\author{
AUTHORS: G. Keller, N. MacLeod, J. B. Lyons and \\ and C. B. Officer
}

\section{APPENDIX includes:}

Figure 1. Site 540 foraminiferal abundance data

Figure 2. Foraminiferal assemblages showing dissolution effects.

Figure 3. Biostratigraphy of DSDP Site 153.

Figure 4. Graphic correlation showing (in-) completeness of Site 536 across the $\mathrm{K} / \mathrm{T}$ boundary and comparison with El Kef (K-T boundary stratotype), Mimbral and Cuba.

Tables 1a, 1b. Percent abundance data of planktic foraminifera at DSDP Site 536.

Table 2. Percent abundance data of planktic foraminifera at DSDP Site 540.

Table 3. Percent abundance data of planktic foraminifera at DSDP Site 95.

DISCUSSION: Nannofossil investigation by James Pospichal. 
DSDP SITE 540

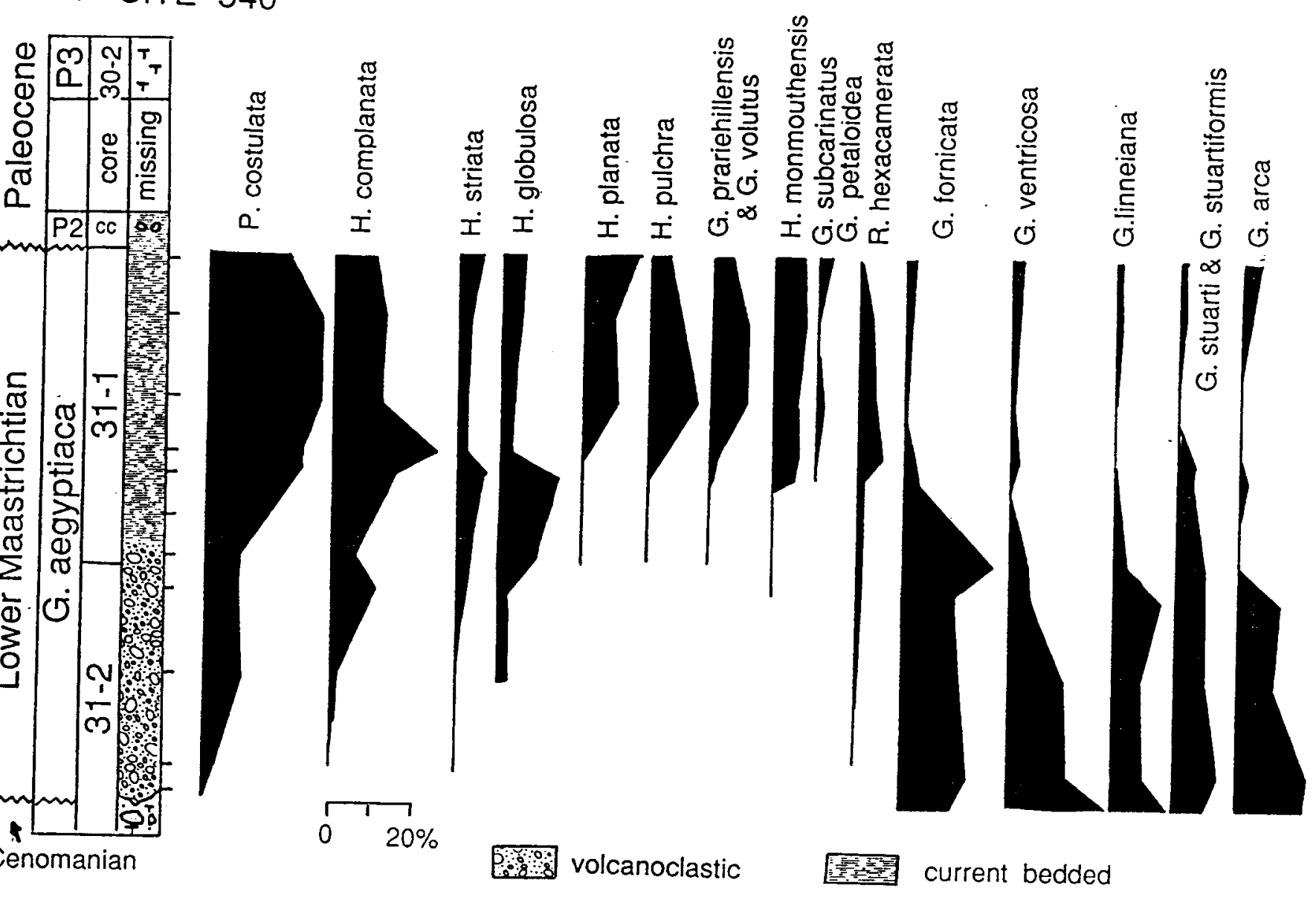

Figure 1. Relative percent abundances of Upper Lower Cretaceous planktic foraminifera in DSDP Site 540, cores 31-1 and 31-2 which directly underlie sediments of Tertiary Zone P2 age. Note, the relative abundance change between core-sections 1 and 2 is due increased carbonate dissolution in the volcanoclastic sediments. This results in increased abundance of dissolution-resistant taxa (globotruncanids). 
DSDP Site 540

Biserial Species

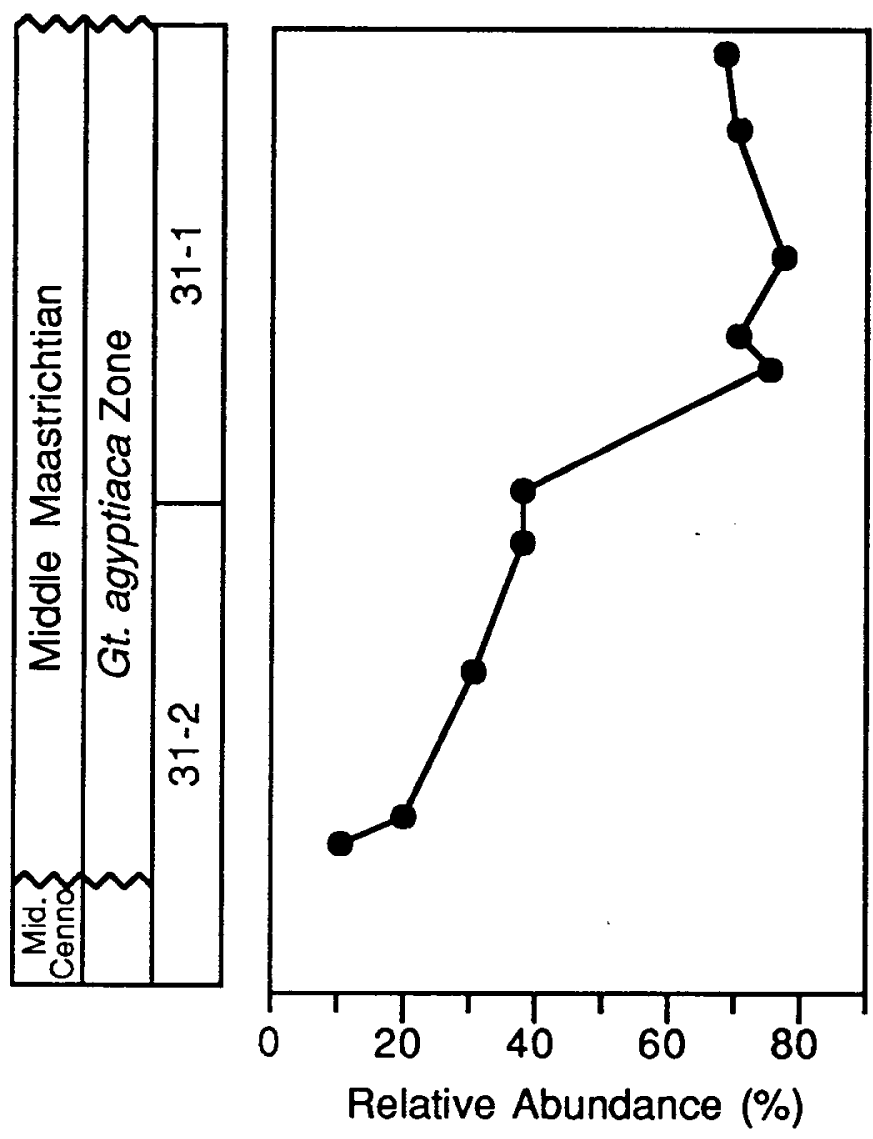

Globotruncanids

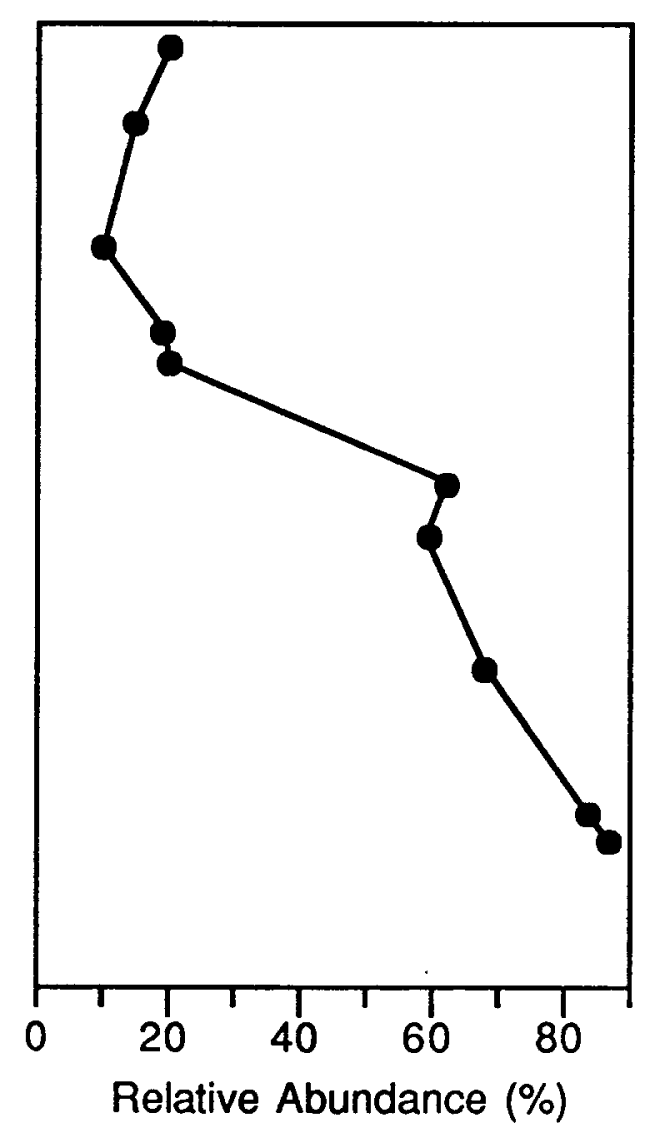

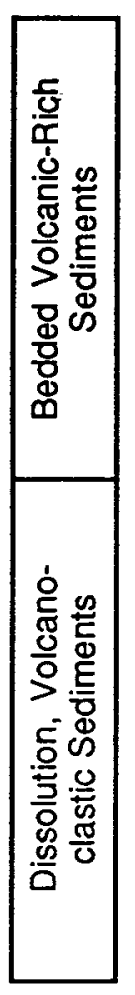

Figure 2 


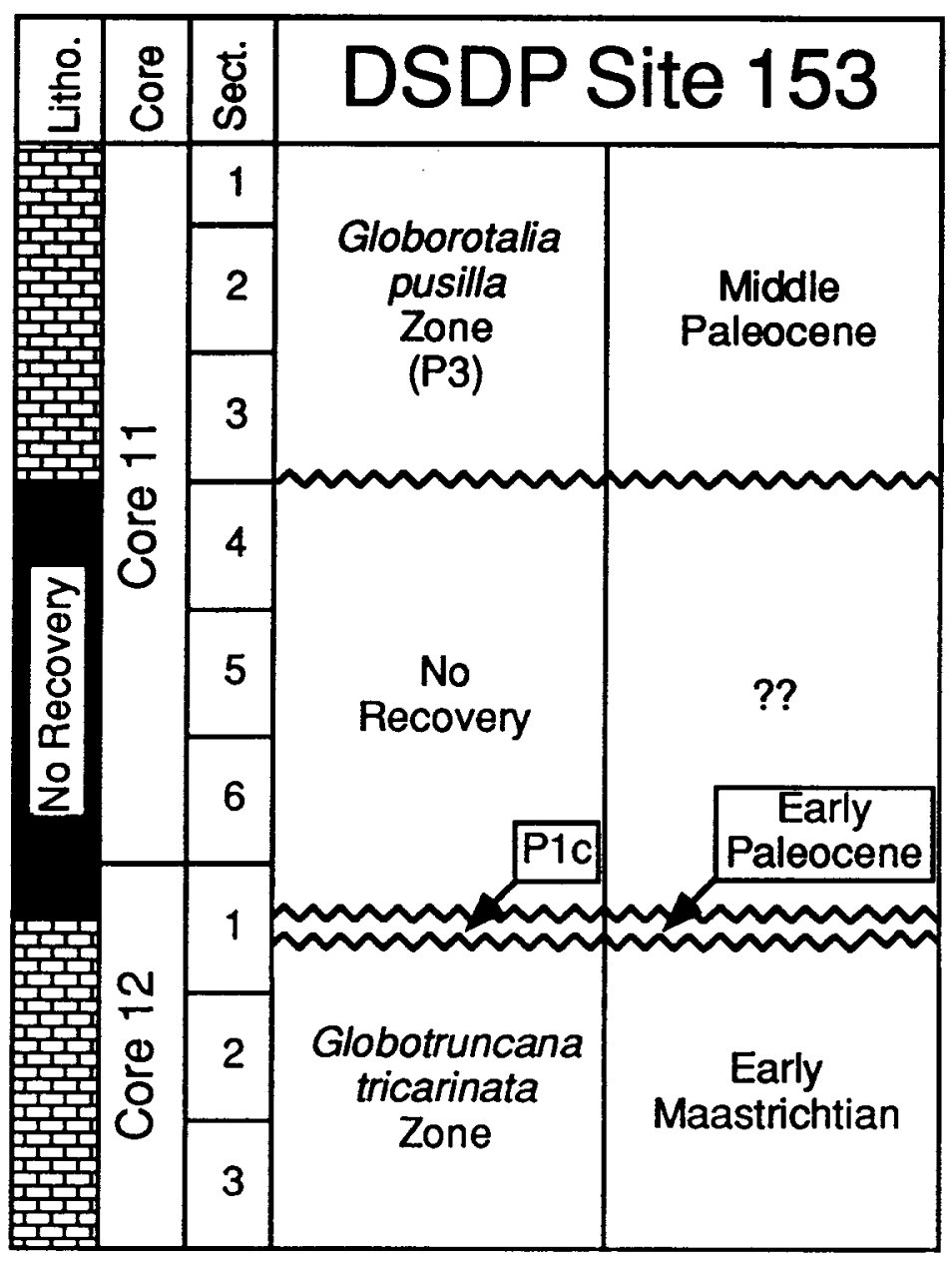

Figure 3 
A. El Kef, Tunisia (Boundary Stratotype)

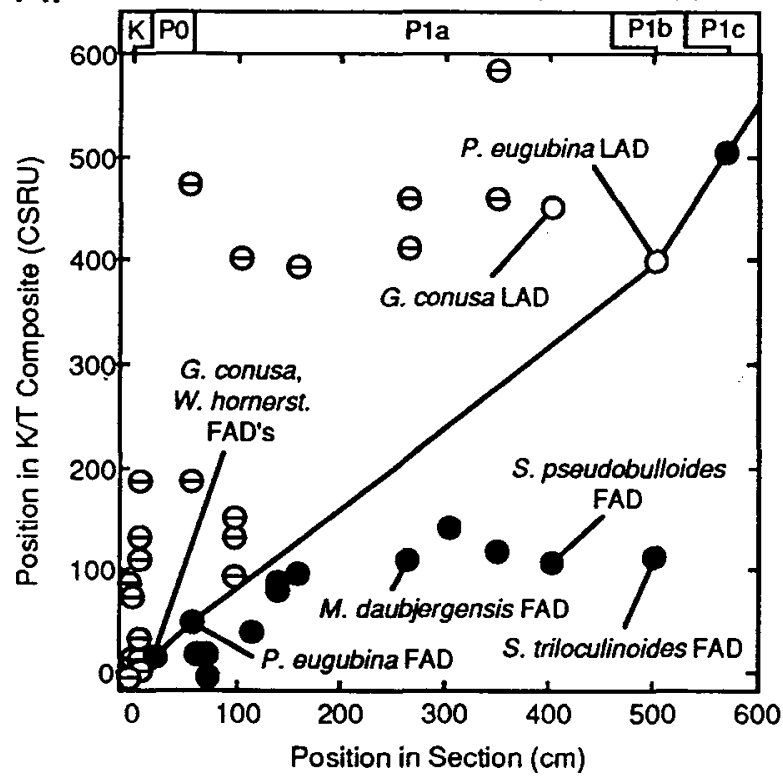

C.

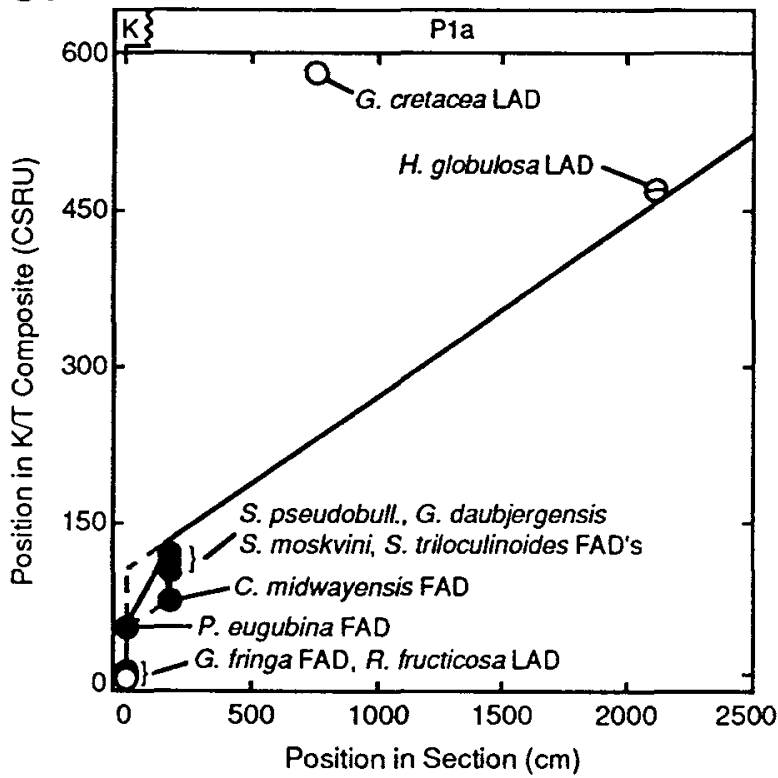

B.

Mimbral, NE Mexico

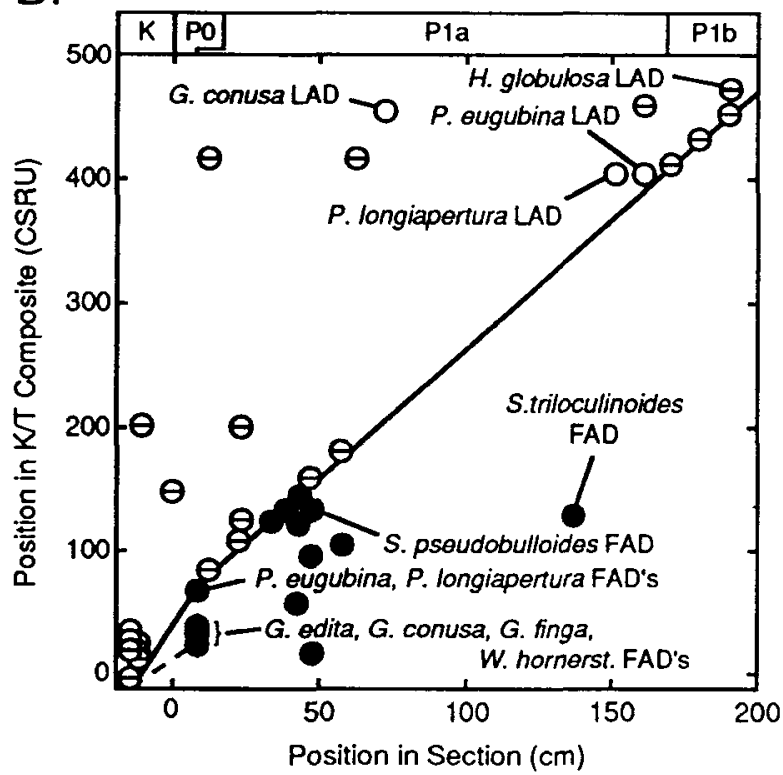

D. DSDP Site 536, Gulf of Mexico

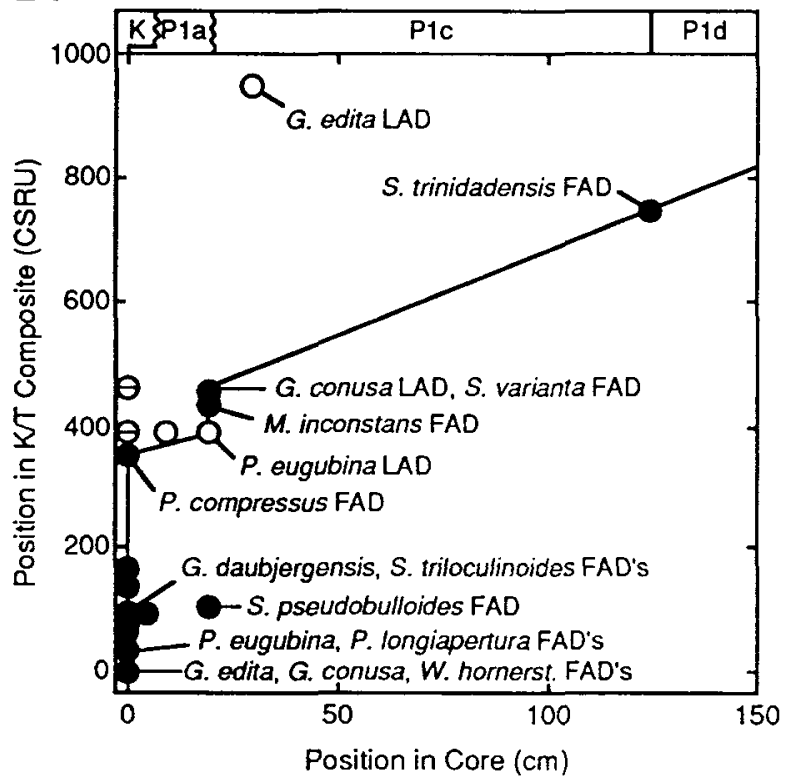

Figure $\$ 4$ 
4

Figure 3 . Graphic correlations with $\mathrm{K} / \mathrm{T}$ composite sections. A: El Kef, Tunisia, B: Mimbral NE Mexico, C: La Alcatraza, Cuba, D: DSDP Site 536. $O=$ last appearance datum of Tertiary taxa, $\theta=$ last appearance datum of Cretaceous survivor taxa, $\bullet=$ first appearance datum. Solid line of correlation represents the interpretation most consistent with biostratigraphic information. Lines of correlation: inclined = interval of net sediment accumulation, vertical $=$ lithological position $(X$ axis) and relative temporal duration ( $Y$ axis) of depositional hiatus. CSRU $=$ composite reference units of MacLeod and Keller (1991a,b). Biostratigraphic zonation above each plot is that of Keller (1988, 1989). 
Table 1a. Relative percent abundance of planktic foraminffera species at DSDP Site 536, Early Paleocene. $x=<1 \%$

\begin{tabular}{|c|c|c|c|c|c|c|c|c|c|c|c|c|c|c|c|c|c|c|}
\hline $\begin{array}{l}\text { Core-section } \\
\text { cm-interval }\end{array}$ & $\begin{array}{l}9-3 \\
100 \\
\end{array}$ & $\begin{array}{r}9-3 \\
126 \\
\end{array}$ & $\begin{array}{l}9-3 \\
145 \\
\end{array}$ & $\begin{array}{l}9-4 \\
15 \\
\end{array}$ & $\begin{array}{l}9-4 \\
35 \\
\end{array}$ & $\begin{array}{l}9-4 \\
55 \\
\end{array}$ & $\begin{array}{l}2-4 \\
75 \\
\end{array}$ & $\begin{array}{l}9-4 \\
96\end{array}$ & $\begin{array}{r}9-4 \\
115 \\
\end{array}$ & $\begin{array}{r}24 \\
145 \\
\end{array}$ & $\begin{array}{l}9-5 \\
10\end{array}$ & $\begin{array}{l}25 \\
20\end{array}$ & $\begin{array}{l}9-5 \\
30\end{array}$ & $\begin{array}{l}9-5 \\
40\end{array}$ & $\begin{array}{l}9-5 \\
50\end{array}$ & $\begin{array}{l}9-5 \\
60 \\
\end{array}$ & $\begin{array}{l}9.5 \\
70\end{array}$ & $\begin{array}{l}9.5 \\
77\end{array}$ \\
\hline Chiloguembelina crinita & $x$ & & & $x$ & & & & & $x$ & & & $x$ & & & & & & \\
\hline Chiloguembelina midwayensis & 2 & 2 & 4 & 7 & 8 & 3 & B & 8 & 5 & 4 & 5 & 5 & 5 & 6 & 3 & $x$ & 4 & $x$ \\
\hline Chibguembelina taurica & & 1 & 2 & 4 & 3 & 1 & 4 & 4 & 5 & 2 & $x$ & 2 & 4 & 1 & 1 & 5 & & \\
\hline Chiloguembelina waparaensis & 18 & 34 & 23 & 12 & 16 & 21 & 18 & 13 & 34 & 20 & 20 & 30 & 17 & 24 & 23 & 5 & 5 & $x$ \\
\hline Woodringina clayloonsis & & 2 & 2 & 2 & 2 & 3 & 2 & 2 & 4 & 4 & 7 & 7 & 5 & 6 & 5 & 4 & $x$ & \\
\hline Woodringina hornerstownensis & $x$ & 5 & 14 & 18 & 14 & 17 & 7 & 16 & 12 & 10 & 15 & 3 & 12 & 10 & 15 & 10 & 2 & 2 \\
\hline Eoolobigerina danica & & & & & & & & & & & & & & & & 2 & & $x$ \\
\hline Eoglobigorina odita & & & & & & & & & & & & & & $x$ & $x$ & 5 & 4 & 6 \\
\hline Eoglobigerina eobulloides & & & & & & & & $x$ & & & & & & $x$ & & 4 & 2 & $x$ \\
\hline Eoglobigerina todina & & & & & & & & & & & & & 1 & & $x$ & 10 & 10 & 3 \\
\hline Eoglobigerina fringa & & & & & & & & & & & & & & & & $\theta$ & 2 & 5 \\
\hline Eoglobigerina trivialis & $x$ & 1 & 1 & $x$ & $x$ & & 1 & $x$ & $x$ & $x$ & $x$ & 1 & 1 & $x$ & & $x$ & $x^{-}$ & \\
\hline Globanomalina pentagona & 1 & 3 & 2 & 4 & 5 & 5 & 6 & 7 & 6 & 6 & $x$ & 6 & 4 & 4 & 4 & 5 & 6 & 4 \\
\hline Globanomalina taurica & & & & & & & & & & & & 1 & & & $x$ & & 1 & \\
\hline Globanomalina totragona & & & & & $x$ & & & & & & & & & & & $x$ & $x$ & \\
\hline Globanomalina polycamera & 2 & $x$ & $x$ & $x$ & 1 & 1 & & $x$ & $x$ & 1 & & & $x$ & $x$ & $x$ & & & \\
\hline Globoconusa conusa & & & & & & & & & & & & & & & & 4 & 3 & $x$ \\
\hline Globoconusa daubjergonsis & $x$ & 1 & $x$ & $x$ & $x$ & $x$ & $x$ & $x$ & 1 & & 1 & $x$ & $x$ & $x$ & $x$ & $x$ & 3 & 7 \\
\hline Guembelitria crotacea & & 2 & & 2 & 4 & 2 & 2 & 7 & & 2 & 14 & $\mathbf{1}$ & 5 & 5 & 4 & 3 & 3 & 2 \\
\hline Guembelitria irregularis & & 1 & & 4 & 5 & 3 & 3 & 3 & $x$ & 6 & 27 & 2 & $x$ & 2 & 2 & $x$ & $x$ & 2 \\
\hline Guembelitria tritolia & & & & & & $x$ & $x$ & & $x$ & $x$ & & $x$ & 1 & 2 & 2 & $x$ & & \\
\hline Igorina spiralis & $x$ & $x$ & & 1 & $x$ & & $x$ & & & & & & & & & & & \\
\hline Morozovella inconstans & 7 & 8 & 15 & 8 & 6 & 4 & 7 & 6 & 3 & 7 & $x$ & 2 & 2 & 2 & 3 & & & \\
\hline Morozovella trinidadensis & 1 & 1 & $\mathfrak{t}$ & 1 & 1 & 1 & 1 & 1 & & & & & & & & & & \\
\hline Murciglobigerina aquiensis & $x$ & & & & & & & & & & & & & & & & & \\
\hline Murciglobigerina chascanona & $x$ & & 2 & $x$ & $x$ & $x$ & $x$ & $x$ & $x$ & 1 & & $x$ & & $x$ & & & & \\
\hline Parvularugoglobigerina ougubina & & & & & & & & & & & & & & & & 23 & 15 & 15 \\
\hline P. eugubina-taurica trans. & & & & & & & & & & & & 2 & $x$ & 8 & 4 & & & \\
\hline Parvularugoglobigerina tongiapertura & & & & & & & & & & & & & & & & 3 & 27 & 40 \\
\hline Planorotalites compressus & 46 & 22 & 20 & 20 & 18 & 22 & 25 & 14 & 14 & 12 & 3 & 10 & 10 & 7 & 7 & 2 & 7 & 5 \\
\hline Subbotina moskvini & 1 & $x$ & 1 & $x$ & 1 & 3 & 1 & 2 & 1 & 4 & 3 & 2 & 5 & 4 & 6 & 1 & & \\
\hline Subbotina psoudobulloides & 6 & • & 7 & 6 & 8 & 8 & 8 & 9 & 6 & 16 & 1 & 18 & 22 & 14 & 14 & & & \\
\hline Subbotina triloculinoides & 3 & 1 & $x$ & 2 & 3 & $x$ & 2 & $x$ & $x$ & & $x$ & 2 & 1 & 1 & 1 & $x$ & $x$ & \\
\hline Subbotina varianta & 5 & 3 & 2 & 4 & & & 1 & 1 & $x$ & 3 & & 2 & & $x$ & $x$ & & & \\
\hline Subbotina triangularis & 2 & & $x$ & $x$ & $x$ & 1 & 1 & $x$ & $\mathbf{x}$ & & & & & & & & & \\
\hline Pseudoguembelina costulata & & & & & & & & & & & & & & & & $x$ & $x$ & $x$ \\
\hline Heterohelix complanata & & & & & & & & & & & & & & $x$ & & & & $x$ \\
\hline Heterohelix globulosa & & & & & & & & & & & & & & $x$ & & & $x$ & $\times$ \\
\hline Total No. specimens counted & 477 & 594 & 595 & 364 & 416 & 416 & 457 & 456 & 432 & 475 & 418 & 443 & 416 & 528 & 449 & 531 & 612 & 333 \\
\hline
\end{tabular}


Table 1b. Relative percent abundances of planktlc foraminiferal species at DSDP Site 536, Late Cretaceous. $x=<1 \%$

\begin{tabular}{|c|c|c|c|c|c|c|}
\hline $\begin{array}{l}\text { Core-section } \\
\text { cm-interval }\end{array}$ & $\begin{array}{l}9-5 \\
80 \\
\end{array}$ & $\begin{array}{l}9-5 \\
90 \\
\end{array}$ & $\begin{array}{r}9-5 \\
100 \\
\end{array}$ & $\begin{array}{r}9-5 \\
110 \\
\end{array}$ & $\begin{array}{r}9-5 \\
120 \\
\end{array}$ & $\begin{array}{r}9-5 \\
140 \\
\end{array}$ \\
\hline Globotruncana egyptiaca & 2 & $x$ & $\mathbf{x}$ & & $x$ & $\vec{q}$ \\
\hline Globotruncana arca & 3 & 2 & $x$ & $x$ & $x$ & \\
\hline Globotruncana bulloides & & & $x$ & & $x$ & 8 \\
\hline Globotruncana duwi & $x$ & & & & & 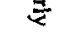 \\
\hline Globotruncana esnehensis & $\mathbf{x}$ & & & & & \\
\hline Globotruncana falsostuarti & & $x$ & 2 & $x$ & 4 & $\bar{z}$ \\
\hline Globotrunçana linneiana & 7 & 5 & 4 & 4 & 7 & 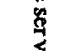 \\
\hline Globotruncana orientalis & $x$ & $x$ & $x$ & 3 & & $\overline{8}$ \\
\hline $\begin{array}{l}\text { Globotruncana rosetta } \\
\text { Globotruncana ventricosa }\end{array}$ & $\begin{array}{l}x \\
x\end{array}$ & $x$ & $x$ & & & $\bar{\Phi}$ \\
\hline Globotruncanita conica & & & & & $x$ & 要 \\
\hline Globotruncanita pettersi & $x$ & & & $x$ & & 总 \\
\hline Globotruncanita stuarti & $x$ & $x$ & $x$ & $x$ & $x$ & $\frac{9}{80}$ \\
\hline Globotruncanita stuartiformis & & $x$ & $x$ & $x$ & $x$ & \\
\hline Rosita fornicata & $x$ & 2 & $x$ & $x$ & $x$ & $\stackrel{m}{Z}$ \\
\hline Rosita patelliformis & 2 & $x$ & $x$ & . & & $\stackrel{\Xi}{\infty}$ \\
\hline $\begin{array}{l}\text { Rosita plummerae } \\
\text { Gansserina cf. gansseri }\end{array}$ & & & & $\mathbf{x}$ & & $\equiv$ \\
\hline Globigerinelloides aspera & 7 & 9 & 7 & 6 & 3 & $\underline{0}$ \\
\hline Globigerinelloides rosebudensis & & & $x$ & $x$ & $x$ & $\xi$ \\
\hline Globigerinelloides subcarinatus & 2 & 4 & 3 & 2 & $x$ & 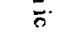 \\
\hline Globigerinelloides volutus & $x$ & & & & & \\
\hline Globotruncanella petaloidea & 2 & $x$ & $x$ & & 4 & $\frac{n}{\tilde{m}}$ \\
\hline Hedbergella holmdelensis & $x$ & $\mathbf{x}$ & 2 & & $x$ & $\underset{n}{\vdots}$ \\
\hline Hedbergella monmouthensis & 6 & 3 & 4 & $x$ & $x$ & \\
\hline Heterohelix complanata & 4 & 4 & 11 & 12 & 9 & $\rightrightarrows$ \\
\hline Heterohelix dentata & $x$ & $x$ & $x$ & $x$ & $x$ & $\tilde{\tilde{\sigma}}$ \\
\hline Heterohelix glabrans & $x$ & $x$ & $x$ & $x$ & & \\
\hline Heterohelix globulosa & 10 & 3 & 6 & 4 & 4 & $\frac{n}{3}$ \\
\hline Heterohelix navarroensis & $x$ & $x$ & $x$ & 2 & $x$ & g \\
\hline Heterohelix planata & & & 2 & 2 & 3 & $\overline{8}$ \\
\hline Heterohelix striata & $x$ & $x$ & $x$ & 2 & 5 & \\
\hline Planoglobulina carseyae & $x$ & $x$ & & & & 喜 \\
\hline Pseudoguembelina costulata & 35 & 45 & 33 & 45 & 32 & \\
\hline Pseudoguembelina excolata & $x$ & $x$ & $x$ & $x$ & 4 & 吾 \\
\hline Pseudoguembelina kempensis & 3 & 3 & 2 & 5 & 2 & $\overline{\underline{\sigma}}$ \\
\hline Pseudoguembelina pulpebra & $x$ & $x$ & $x$ & $x$ & $x$ & $\frac{\xi}{2}$. \\
\hline Pseudoguembelina punctulata & $x$ & $x$ & & & $x$ & \\
\hline Pseudotextularia deformis & & & $x$ & & & \\
\hline Pseudotextularia elegans & $\mathbf{x}$ & $\mathbf{x}$ & $\mathbf{x}$ & $x$ & $x$ & \\
\hline Gublerina robusta & & & & & $x$ & \\
\hline Racemiguembelina fructicosa & $x$ & & & & & \\
\hline Racemiguembelina powelli & & $x$ & & & & \\
\hline Rugoglobigerina hexacamerata & 4 & 5 & 6 & 4 & 5 & \\
\hline Rugoglobigerina rugosa & 2 & $x$ & $x$ & $x$ & $x$ & \\
\hline Rugoglobigerina scotti & $x$ & & $x$ & $x$ & $x$ & \\
\hline Ventrilabella manneloensis & $x$ & & & & & \\
\hline Ventrilabella multicamerata & $\mathbf{x}$ & & & & & \\
\hline Total No. of specimens counted & 216 & 273 & 274 & 237 & 240 & \\
\hline
\end{tabular}


Table 2. Planktic foraminiferal species and number of specimens present in $5 \mathrm{ch}$ centimeters of sediment per sample at DSDP Site 540, Late Cretaceous.

Preservation is poor with strong dissolution in predominantly volcanic sediments.

\begin{tabular}{|c|c|c|c|c|c|c|c|c|c|c|c|}
\hline $\begin{array}{l}\text { Core-section } \\
\text { cm-interval }\end{array}$ & $\begin{array}{c}31-1 \\
8 \\
\end{array}$ & $\begin{array}{c}31-1 \\
30 \\
\end{array}$ & $\begin{array}{c}31-1 \\
70 \\
\end{array}$ & $\begin{array}{c}31-1 \\
95 \\
\end{array}$ & $\begin{array}{l}31-1 \\
105 \\
\end{array}$ & $\begin{array}{l}31-1 \\
125 \\
\end{array}$ & $\begin{array}{l}31-1 \\
145 \\
\end{array}$ & $\begin{array}{c}31-2 \\
10 \\
\end{array}$ & $\begin{array}{c}31-2 \\
50 \\
\end{array}$ & $\begin{array}{c}31-2 \\
95 \\
\end{array}$ & $\begin{array}{l}31-2 \\
105 \\
\end{array}$ \\
\hline Globotruncana egyptiaca & & & 4 & 1 & & & & 3 & & & \\
\hline Globotruncana arca & 1 & 6 & & 3 & 3 & 1 & 10 & 11 & & & 6 \\
\hline Globotruncana bulloides & 3 & 5 & 6 & & & & 5 & 8 & 5 & & \\
\hline Globotruncana falsostuarti & 2 & 6 & 3 & 3 & 12 & & 13 & 5 & 8 & 1 & 7 \\
\hline Globotruncana linneiana & 4 & 4 & & & 4 & & 4 & 12 & 10 & 3 & 6 \\
\hline Globotruncana orientalis & 7 & & & 2 & & & 2 & 1 & 7 & 7 & 2 \\
\hline Globotruncana rosetta & & & & & & & & & 1 & 1 & 1 \\
\hline Globotruncana ventricosa & & & 1 & & 1 & & 7 & & 3 & 5 & \\
\hline Globotruncanita angulata & 2 & & & & & & & & & & \\
\hline Globotruncanita conica & & & & & & & & & 2 & 1 & \\
\hline Globotruncanita stuarti & 1 & 3 & & & 2 & & 6 & & & & \\
\hline Globotruncanita stuartiformis & 2 & 1 & & 3 & & & 2 & 7 & 6 & 4 & 3 \\
\hline Rosita fornicata & 3 & 4 & 1 & 2 & 3 & 1 & 26 & 6 & 15 & 2 & 4 \\
\hline Rosita patelliformis & 4 & 1 & & & 5 & & 1 & 8 & 5 & 5 & 1 \\
\hline Rosita plummerae & & & & & 1 & & 2 & & 11 & & \\
\hline Gansserina of. gansseri & 1 & & & & & & & & & & \\
\hline Gansserina wiedenmayeri & & & & & & & & & & 2 & \\
\hline Globigerinelloides aspera & 7 & 13 & 9 & 1 & 4 & & 1 & & & & \\
\hline Globigerinelloides subcarinatus & & 2 & 3 & & & & & & & & \\
\hline Globigerinelloides volutus & 2 & 6 & 14 & 1 & 1 & & & & & & \\
\hline Globotruncanella petaloidea & 6 & & 2 & & 2 & & & 1 & & & \\
\hline Hedbergella monmouthensis & 2 & 13 & 10 & 1 & 8 & & & 1 & & & \\
\hline Hedbergella holmdelensis & 9 & 4 & 3 & 1 & 2 & & & & & & \\
\hline Heterohelix complanata & 18 & 29 & 29 & 20 & 28 & & 9 & 12 & 3 & 1 & \\
\hline Heterohelix dentata & 9 & & 2 & & 1 & & & & & & \\
\hline Heterohelix glabrans & 10 & 15 & 29 & 1 & 1 & & 3 & & & & \\
\hline Heterohelix globulosa & 10 & 9 & 8 & 5 & 26 & & 12 & 3 & 5 & & \\
\hline Heterohelix navarroensis & 3 & 3 & 19 & 1 & 3 & & & & & & \\
\hline Heterohelix planata & 25 & 13 & 22 & & 1 & & 2 & & & & \\
\hline Heterohelix pseudotessera & & & 1 & & & & & & & & \\
\hline Heterohelix striata & 8 & 7 & 4 & 2 & 11 & & 5 & 4 & 2 & 1 & \\
\hline Planoglobulina carseyae & & 1 & & & & & & & & & \\
\hline Pseudoguembelina costulata & 38 & 61 & 70 & 17 & 41 & 1 & 11 & 8 & 10 & 2 & 1 \\
\hline Pseudoguembelina excolata & & 2 & 1 & & 1 & & & 1 & 2 & & \\
\hline Pseudoguembelina kempensis & & & & 2 & 6 & & & 1 & & & \\
\hline Pseudoguembelina pulpebra & & 2 & 1 & 1 & 3 & & 3 & 1 & 5 & 1 & 3 \\
\hline Pseudotextularia deformis & & & & & 3 & & & 4 & 4 & & \\
\hline Pseudotextularia elegans & 1 & 3 & 1 & 1 & 5 & & 3 & 6 & 3 & 3 & \\
\hline Gublerina robusta & 1 & 1 & & 1 & & & & & & & \\
\hline Rugoglobigerina hexacamerata & 2 & 8 & 10 & 5 & 5 & & 4 & 3 & 3 & 1 & \\
\hline Rugoglobigerina rugosa & & & & & & & & & 1 & 2 & 1 \\
\hline Ventrilabella multicamerata & & & & 1 & 1 & & 1 & & & & \\
\hline Archeoglobigerina blowi & & & & & & & & & 2 & 3 & 2 \\
\hline Total No. of specimens counted & 182 & 224 & 255 & 75 & 184 & 3 & 132 & 106 & 111 & 42 & 35 \\
\hline
\end{tabular}


Table 3. Relative percent abundance of planktic Foraminifera at DSDP Site 95, Paleocene. $x=<1 \%$

\begin{tabular}{|c|c|c|c|c|c|c|c|}
\hline $\begin{array}{l}\text { Core-section } \\
\mathrm{cm} \text {-interval }\end{array}$ & $\begin{array}{c}13-1 \\
80 \\
\end{array}$ & $\begin{array}{l}13-1 \\
111 \\
\end{array}$ & $\begin{array}{c}13-1 \\
128 \\
\end{array}$ & $\begin{array}{c}13-1 \\
141 \\
\end{array}$ & $\begin{array}{c}13-2 \\
3 \\
\end{array}$ & $\begin{array}{c}13-2 \\
15 \\
\end{array}$ & $\begin{array}{c}13-2 \\
23 \\
\end{array}$ \\
\hline Chiloguembelina midwayensis & $x$ & & & & & & $x$ \\
\hline Chiloguembelina waiparaensis & $\mathrm{x}$ & 4 & & & & & \\
\hline Woodringina claytoensis & 8 & 8 & & & 2 & 2 & $x$ \\
\hline Woodringina hornerslownensis & 17 & 27 & & & 70 & 77 & 77 \\
\hline Eoglobigerina danica & & & & & 13 & 12 & 9 \\
\hline Eoglobigerina edita & & & & & & & \\
\hline Eoglobigerina eobulloides & 2 & & & & & & \\
\hline Eoglobigerina fodina & & & & & & $x$ & $x$ \\
\hline Eoglobigerina simplicissima & 3 & $x$ & & & $x$ & $x$ & $x$ \\
\hline Globanomalina pentagona & $x$ & & & & $x$ & $x$ & \\
\hline Globanomalina taurica & $x$ & & & & $x$ & & $x$ \\
\hline G. eugubina-taurica trans. & & $x$ & & & & $x$ & \\
\hline Globoconusa conusa & $x$ & $x$ & & & $x$ & & $x$ \\
\hline Globoconusa daubjergensis & $x$ & & & & & & \\
\hline Guembelitria cretacea & 45 & 39 & & & $x$ & 3 & $x$ \\
\hline Guembelitria danica & 6 & 2 & & & $x$ & $x$ & $x$ \\
\hline Guembelitria irregularis & 8 & 9 & & & & & \\
\hline Guembelitria trifolia & 4 & $x$ & & & & & \\
\hline Parvularugoglob. eugubina & & & & & 5 & $x$ & 8 \\
\hline P. longiapertura & & & & & & & $x$ \\
\hline Planorotalites compressus & $x$ & $x$ & & & $x$ & & \\
\hline Subbotina moskvini & & $x$ & & & & & \\
\hline Subbotina pseudobulloides & $x$ & $x$ & & & $x$ & & $x$ \\
\hline Subbotina triloculinoides & $x$ & & & & & & \\
\hline Pseudoguembelina costulata & $x$ & & & & & & \\
\hline Globigerinelloides aspera & & $x$ & & & $x$ & $x$ & \\
\hline Total No. specimens counted & 386 & 278 & dissol. & dissol. & 323 & 307 & \\
\hline Zone & P1c & Pic & Hiatus & Hiatus & P1a & P1a & P1a \\
\hline
\end{tabular}

Also examined: $12-4,72 \mathrm{~cm}, 12-4,144 \mathrm{~cm}, 13-1,63 \mathrm{~cm}$, Danian assemblage Zone P1c.

$13-2,106 \mathrm{~cm}$, dissolution, mostly benthics present. $13-3,100 \mathrm{~cm}$, Middle Maastrichtian

G. aegyptiaca Zone (G. conica, G. arca, G. stuarti, G. stuartiformis, R. fomicata,

$G$. ventricosa, $G$. linneiana, $G$. havanensis, $H$. carinata, $V$. multicamerata,

$G$. aspera, Gublerina robusta, H. dentata, $P$. elegans.) 


\section{Nannofossil investigation by James Pospichal}

Nannofossils may suggest a younger upper Maastrichtian age, although they also indicate that the latest Maastrichtian is missing. Calcareous nannoplankton in the Maastrichtian sediments of Sites 536 and 540 are poorly preserved with calcite overgrowths complicating positive identification of species. Nevertheless, reexamination of the Maastrichtian interval of Site 536 (core 9-5, 90-140 cm) by James Pospichal indicates the presence of rare Micula prinsii in sample $9-5,90 \mathrm{~cm}$ and few to common $M$ : murus and common Lithaphridites quadratus in the $50 \mathrm{~cm}$ interval below (J. Pospichal, 1993, written commun.). Rare to few reworked lower Maastrichtian and Campanian species are also present. Preservation in Tertiary samples and the topmost Maastrichtian sample $(9-5,80 \mathrm{~cm})$ at Site 536 is too poor for positive identification.

Micula prinsii, the uppermost Maastrichtian index taxon (M.prinsii Zone), is short ranging and morphologically intergrades with ancestral $M$. murus (the index taxon of the preceding zone). Since the Site $536 \mathrm{M}$. prinsil populations are confined to one sample near the the top of the Maastrichtian sediments and resemble early transitional morphotypes, this suggests that at least the top of the Maastrichtian is missing. At Site 540 (cores 31-1 to 31-2), M. murus and L. quadratus are also present, but no $M$. prinsii were found. This suggest that this interval is older than Site $536(9-5,90-140 \mathrm{~cm})$ and no younger than the $M$. murus Zone.

The age discrepancy between the foraminiferal and nannofossil biostratigraphies may be largely due to the poor nannofossil preservation and carbonate dissolution. Despite this discrepancy, however, both microfossil groups indicate that at least the uppermost Maastrichtian (M.prinsii Zone in Site 540) is missing. Alvarez et al.'s (1992) claim that the depositional age of the Maastrichtian sediments of Sites 536 and 540 "cannot be distinguished from the age of the K-T boundary" (p. 698) is, therefore, incorrect. 